\title{
Hipoacusia súbita. Experiencia de un año
}

\section{Sudden hearing loss. A one-year experience}

Jorge Zúñiga P¹, Carolina Espinoza G², Natalia Tamblay³, Cristián Martínez².

\section{RESUMEN}

Introducción: La hipoacusia súbita es un cuadro clínico que presenta controversias tanto en su etiología, como en su tratamiento. Existen escasos estudios en la literatura nacional sobre este tema.

Objetivo: Evaluar la incidencia del cuadro de hipoacusia súbita en un año, en el Hospital Cínico de la Universidad de Chile, analizar características epidemiológicas, clínicas, y del tratamiento.

Material y método: Estudio retrospectivo realizado en el Hospital Cínico de la Universidad de Chile, revisando fichas clínicas entre Diciembre del año 2004 hasta Noviembre de 2005, con diagnóstico de hipoacusia súbita

Resultados: Se realizó el estudio con 43 pacientes con diagnóstico de hipoacusia súbita La mayoría recibió tratamiento corticoidal. $\mathrm{\theta} 28 \%$ de los pacientes mejoraron, el 18,7\% tuvo una recuperación parcial, 9,3\% recuperación total, no presentaron mejoría el 72\%. Se encontró asociación entre menor edad y mejoría en forma casi significativa Se encontró una asociación entre mejoría y compromiso del oído derecho en forma significativa

Conclusión: La mayoría de los hallazgos son comparables con la literatura nacional e internacional, la asociación mejoría v/s oído derecho debe ser validada por nuevos estudios.

Palabras clave: Hipoacusia súbita, sordera súbita, corticoides, factores de riesgo.

\section{ABSTRACT}

Introduction. Sudden hearing loss (SHL) is a medical condition somewhat controversial in its etiology and treatment. There are few reports of $\mathrm{SH}$ in the local literature.

Aim. To assess the incidence of SHL at the University of Chile Hospital during a year-long period, analyzing the epidemiological, clinical and treatment characteristics.

Material and Method. Restrospective review of SHL clinical data between December 2004 and November 2005.

Results. Forty three patients diagnosed with SHL were included. Most were treated with corticoids. In $28 \%$ of cases, there was an improvement of the condition. O these, in $18.7 \%$ there was a partial improvement, and in 9.3\% a complete recovery. There was no improvement in $72 \%$ of the cases. The relation between age and recovery was almost significant. A significant relation between improvement and right ear involvement was found.

Conclusion. Most of our findings are similar to those reported in the literature; the association between recovery an right ear involvement should be validated by future studies.

Keywords. Sudden hearing loss, sudden deafness, corticoids, risk factors.

\footnotetext{
Médico del Servicio de Otorrinolaringología del Hospital Clínico de la Universidad de Chile

2 Programa de Anatomía y Biología del Desarrollo - ICBM - Facultad de Medicina Universidad de Chile

3 Interna de Medicina, Universidad de Chile

4 Tecnólogo Médico del Servicio de Otorrinolaringología del Hospital Clínico de la Universidad de Chile
} 


\section{INTRODUCCIÓN}

La sordera o hipoacusia súbita fue descrita por primera vez en 1944 por De Kleyn 1 .

Se define como: hipoacusia sensorioneural, mayor a $30 \mathrm{~dB}$ de pérdida en al menos tres frecuencias consecutivas, que se desarrolla en un periodo de horas hasta 3 días. Debe diferenciarse de la sordera rápidamente progresiva que es la pérdida auditiva que ocurre en un periodo superior a 72 horas 2,3 .

La mayoría de los casos son idiopáticos, en sólo un $10-15 \%$ se descubre la etiología 4 .

Su incidencia es desde 5 a 20 por 100.000 habitantes, afecta por igual a ambos sexos, la mayoría de los casos ocurren en la $5^{\circ}$ década de la vida, habitualmente unilateral, un $2 \%$ puede ser bilateral ${ }^{5}$.

\section{Etiología}

La etiología de este cuadro es difícil de precisar, se han descrito diferentes teorías que intentan precisarla. Entre éstas destacan: viral, vascular, ruptura de membranas, e inmunológica ${ }^{3}$.

Se ha encontrado que entre un 25 y un $40 \%$ de los pacientes que sufren esta patología refieren el antecedente de infección respiratoria alta dentro del mes anterior al cuadro clínico de sordera súbita. Se han relacionado con este cuadro infecciones por virus parotiditis, rubéola, herpes zoster, herpes simplex, citomegalovirus e influenza $B^{6-8}$. Además, existen los trabajos clásicos de Schuknecht y Yoon, de histopatología del hueso temporal que mostrarían asociación de la sordera súbita con la infección viral9,10.

La teoría vascular postula que la falta de un aporte sanguíneo a la cóclea produciría "infartos cocleovestibulares", el daño vascular sería obstructivo (hiperviscosidad, microangiopatías, infartos cocleovestibulares, vasoespasmo) 0 hemorrágico ${ }^{12-14}$.

La teoría inmunológica se basa en que el oído sería el órgano diana de enfermedades inmunomediadas como: colitis ulcerosa, policondritis recidivante, lupus eritematoso sistémico, poliarteritis nodosa, granulomatosis de Wegener. Podría existir además una autoinmunidad inducida por antígenos virales, que asociaría esta teoría con la teoría vira|15-17.
La ruptura de la membrana de Reissner (en el hídrops) 0 de las ventanas oval y redonda podrían originar también un cuadro de sordera súbita ${ }^{18}$.

\section{Cuadro clínico}

En cuanto a la clínica se dice que habitualmente la pérdida auditiva se percibe en la mañana, se asocia a esfuerzo físico o emocional. Los síntomas más frecuentes son la sensación de oído tapado y tinnitus, un $40 \%$ de los pacientes relata mareos y vértigo y un $25 \%$ presenta cofosis ${ }^{19}$.

Se han reconocido algunos factores predisponentes como: edad avanzada, cambios de altitud y presión atmosférica, variaciones climáticas, consumo excesivo de alcohol, diabetes mellitus, arteriosclerosis, embarazo, uso de anticonceptivos orales, estrés quirúrgico y anestesia genera ${ }^{20}$.

Dos tercios de los pacientes se recuperan espontáneamente en las 2 semanas siguientes (1/3 total, $1 / 3$ parcial), mientras un tercio de los casos no se recuperan ${ }^{21}$.

Son factores asociados a mal pronóstico: edad avanzada, hipoacusias profundas con gran compromiso de los agudos y accidente cocleovestibular $22-24$.

\section{Estudio}

En el estudio de la hipoacusia súbita deben solicitarse: audiometría, examen vestibular, hemograma-VHS, VDRL o FTA-ABS, perfil lipídico, uremia, glicemia, HIV y títulos antivirales (parotiditis, rubéola, herpes simplex, citomegalovirus e influenza B) (Tabla 1).

\section{Tabla 1. Estudio sugerido de hipoacusia súbita}
1. Historia clínica y examen $\mathrm{ORL}$
2. Audiometría
3. Examen vestibular
4. Hemograma-VHS
5. VDRL - FTA-ABS
6. Perfil lipídico (triglicéridos)
7. Glicemia/Uremia
8. VIH
9. Títulos antivirales
10. RNM 
En cuanto a exámenes imagenológicos la tomografia computada no ha demostrado ser útil, la resonancia nuclear magnética de cerebro y ángulo pontocerebeloso es útil en el diagnóstico de tumores pontocerebelosos y enfermedades autoinmunes, además permite observar focos de isquemia y signos sugerentes de esclerosis múltiple ${ }^{15,25}$.

\section{Tratamiento}

Es controversial. Se han usado vasodilatadores desde 1919 (Lermoyes), algunos de ellos son: histamina, ácido nicotínico, papaverina, procaína, niacina, y carbógeno. Se ha demostrado que los vasodilatadores no tienen efecto sobre el flujo sanguíneo coclear26-28.

Los anticoagulantes fueron propuestos por Fowler en 1957, algunos son: heparina, coumadin, pentoxifilina y dextran. Actualmente poco utilizados por sus severos efectos secundarios ${ }^{29}$.

Trombolíticos como desfibrogenasa y estreptoquinasa no han tenido resultados significativos ${ }^{30}$.

Antivirales han sido utilizados (aciclovir, amantadina) contra el virus herpes simplex. Han demostrado eficacia terapéutica al asociarse a corticoides $^{6}$, sin embargo, nuevos estudios no han encontrado diferencias significativas al placebo ${ }^{7}$.

La utilización de corticoides fue propuesta por Hilger en 1950, posteriormente Wilson demostró que éstos son útiles en pacientes con perdidas auditivas entre los 40 y $90 \mathrm{~dB}$ y no tienen efecto en pacientes con pérdidas $>90 \mathrm{~dB}$. Moskowitz encontró similares resultados ${ }^{31,32}$.

Existen cientos de estudios sobre la eficacia de los corticoides en la hipoacusia súbita. Una revisión Cochrane estudió 516 resúmenes de estudios de efectividad clínica de los corticoides, de éstos separó 30 de mejor calidad metodológica y por último eligió dos trabajos: Cinamon $2001^{33}$ y Wilson $1980^{31}$. La conclusión final de este estudio fue que ambos ensayos fueron de mala calidad metodológica, uno mostró un beneficio de los corticoides y el otro $\mathrm{no}^{34}$.

Dado lo controversial del tema decidimos realizar un estudio retrospectivo de un año de duración de los casos de sordera súbita vistos en nuestro servicio.

\section{OBJETIVO}

Nuestro objetivo fue evaluar la incidencia del cuadro de hipoacusia súbita en un año, en el Hospital Clínico de la Universidad de Chile, analizar características epidemiológicas, clínicas, y del tratamiento.

\section{MATERIAL Y MÉTODO}

Se realiza un estudio retrospectivo de todos los pacientes con diagnóstico de sordera súbita, hipoacusia súbita, y accidente cocleovestibular, vistos por primera vez en el Servicio de Otorrinolaringología del Hospital Clínico de la Universidad de Chile, desde Diciembre del año 2004 hasta Noviembre del año 2005.

Se constata edad de los pacientes, sexo, antecedentes mórbidos previos, oído afectado, actividad que realizaba en el momento de constatar la hipoacusia, síntomas asociados, tiempo de demora en la consulta, incidencia estacional, tipo de audiometrías, estudio realizado, tratamiento realizado y resultados del tratamiento.

En el análisis de datos, se realizó primero una caracterización de la muestra, para lo cual se estimó porcentajes para las variables dicotómicas, y promedio para la variable edad. Para evaluar asociación entre las variables se utilizó primero el test de proporción para las variables dicotómicas y $\mathrm{t}$ student para las variables discretas. Para la determinación de factores de riesgo y de protección, ajustando por el resto de las variables, se realizó una regresión logística multivariada, se consideró significativo un valor $\mathrm{p}<0,05$.

Para el análisis estadístico de las variables categóricas no dicotómicas (tipo de curva v/s mejoría) se realizó una prueba de Fisher.

\section{RESULTADOS}

Se encontraron 51 pacientes con diagnóstico de sordera súbita 0 hipoacusia súbita, fueron descartados 8 pacientes que presentaban un diagnóstico erróneo: uno por presentar una hipoacusia por trauma acústico agudo, dos con diagnóstico de hipoacusia autoinmune, cinco con diagnóstico de 
hídrops. En total se realizó el estudio con 43 pacientes con diagnóstico de hipoacusia súbita.

Un $53,5 \%$ de los pacientes (23 pacientes) fue de sexo femenino contra un $46,51 \%$ (20 pacientes) de sexo masculino.

El 53,5\% (23 pacientes) presentó el cuadro en su oído izquierdo, 46,5\% (20 pacientes) en el oído derecho. No hubo pacientes con afectación bilateral.

La mayor parte de los pacientes se concentró entre la quinta y séptima década de la vida (18 pacientes entre los 41 y 60 años). El menor tuvo 13 años y el mayor 88 años (Figura 1). La edad media fue de 51 años.

En cuanto a antecedentes mórbidos lo más frecuente fue Hipertensión Arterial (8 pacientes), Diabetes Mellitus tipo II (4 pacientes) y atopía (3 pacientes) (Tabla 2).

Los medicamentos que estaban consumiendo los pacientes en el momento del cuadro se ilustran en la Tabla 3.

El momento del inicio del cuadro lo relatan claramente 13 pacientes: en la noche (3 pacientes), en la madrugada (2 pacientes), acostados ( 3 pacientes), al levantarse (3 pacientes), después de mala noticia (1 paciente), después del baño (1 paciente).

Tres pacientes relacionaron el cuadro de hipoacusia con un cuadro de infección respiratoria alta previo.

En cuanto a incidencia estacional del cuadro: 13 pacientes en primavera, 10 pacientes en verano, 10 pacientes en otoño y 10 pacientes en invierno (Figura 2).

A cuatro pacientes se les indicó hospitalización por presentar Diabetes Mellitus II, un paciente rechazó la hospitalización y el tratamiento.

El síntoma más frecuente asociado a la hipoacusia fue el tinnitus en 32 pacientes y el vértigo en 15 pacientes (Tabla 4).

La mayoría de los pacientes consultaron antes de los 15 días de evolución de su cuadro, sólo 12 consultaron antes de las 72 horas (27,9\%) (Figura 3).

En el estudio audiométrico se constató una mayor proporción de curva descendente (44,2\%, 19 pacientes), curva plana en $23,3 \%$, curva ascendente en un $7 \%$ y cofosis se encontró en un $25 \%$.
Se hizo estudio funcional de VIII par (cocleovestibular) a 15 pacientes, de ellos 9 presentaron compromiso vestibular del mismo lado de la hipoacusia.

Se realizó Tomografía computada cerebral en 10 pacientes, resonancia nuclear magnética con gadolinio en 10 pacientes, angiotac en 1 paciente.

En un paciente fue diagnosticado un meningioma petroclival.

\section{Tabla 2. Antecedentes mórbidos}

\begin{tabular}{|lc|}
\hline Antecedente & N pacientes \\
\hline Hipertensión arterial & 8 \\
Diabetes mellitus & 4 \\
Atopia & 3 \\
Dislipidemia & 2 \\
Asma bronquial & 2 \\
Arritmia & 2 \\
Consumo excesivo de alcohol & 2 \\
Quirúrgicos & 2 \\
Otitis media fibroadhesiva & 1 \\
Nefrolitiasis & 1 \\
Glaucoma & 1 \\
Sordera súbita & 1 \\
Artritis reumatoídea & 1 \\
Hipotiroidismo & 1 \\
Estrés emocional & 1 \\
Cáncer gástrico & 1 \\
Gota & 1 \\
Exposición a ruidos & 1 \\
\hline
\end{tabular}

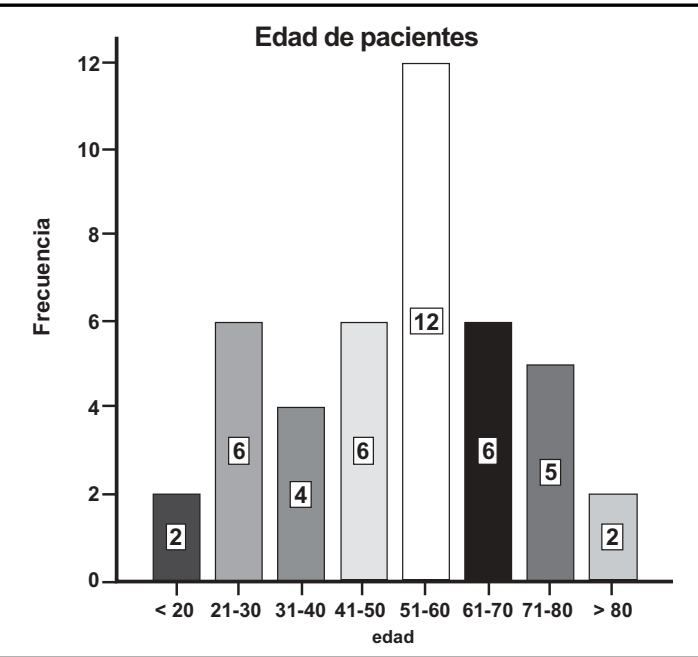

Figura 1. Distribución de los pacientes según su edad. 
Tabla 3. Medicamentos que utilizaban los pacientes en el momento del cuadro

\begin{tabular}{|lc|}
\hline Fármaco & N pacientes \\
\hline Glibenclamida & 2 \\
Metformina & 2 \\
Omeprazol & 1 \\
Bromuro de ipatropio & 1 \\
Hidroclorotiazida + Triamterene & 1 \\
Betahistina diclorohidrato & 1 \\
Atenolol & 1 \\
Amiodarona & 1 \\
Terapia de reemplazo hormonal & 1 \\
Colchicina & 1 \\
Losartán & 1 \\
Digoxina & 1 \\
Diltiazem & 1 \\
Acenocumarol & 1 \\
Paracetamol & 1 \\
Cisaprida & 1 \\
Oticum & 1 \\
\hline
\end{tabular}

A 32 pacientes se les indicó tratamiento corticoidal, uno rechazó el tratamiento. La mayoría fueron tratados con prednisona $1 \mathrm{mg} / \mathrm{kg} /$ día.

Diez pacientes fueron tratados con vasodilatadores, 1 paciente con antiagregante plaquetario y 3 pacientes con otros tratamientos coadyuvantes. Once pacientes no fueron tratados con corticoides porque presentaban más de dos meses de evolución, y sólo tuvieron tratamiento con vasodilatadores o no se les entregó tratamiento (Tabla 5).

El $28 \%$ de los pacientes mejoraron, el 18,7\% tuvo una recuperación parcial (8 pacientes), 9,3\% recuperación total (4 pacientes), no presentaron mejoría el $72 \%$ (31 pacientes) (Tabla 6).

La edad de los pacientes se asoció a la mejoría en forma casi significativa ( $p$ value $0,05)$, los pacientes que mejoraron tenían un promedio de edad de 42 años, los que no mejoraron un promedio de 54 años.

El sexo no se asoció a una probabilidad de mejorarse.

Aquellos pacientes que tuvieron un compromiso del OD mejoraron un $45 \%$, aquellos en que el compromiso era en Ol mejoraron un $13 \%$, diferencia que fue estadísticamente significativa ( $p$ value 0,01 ).

No se encontró diferencia estadísticamente significativa en la mejoría de los pacientes que
Tabla 4. Síntomas asociados por pacientes, consignados en las fichas clínicas

\begin{tabular}{|lc|}
\hline Síntoma & N pacientes \\
\hline Tinnitus & 32 \\
Vértigo & 15 \\
Náuseas & 7 \\
Vómitos & 7 \\
Mareos & 2 \\
Autofonía & 1 \\
Parálisis facial contralateral & 1 \\
\hline
\end{tabular}

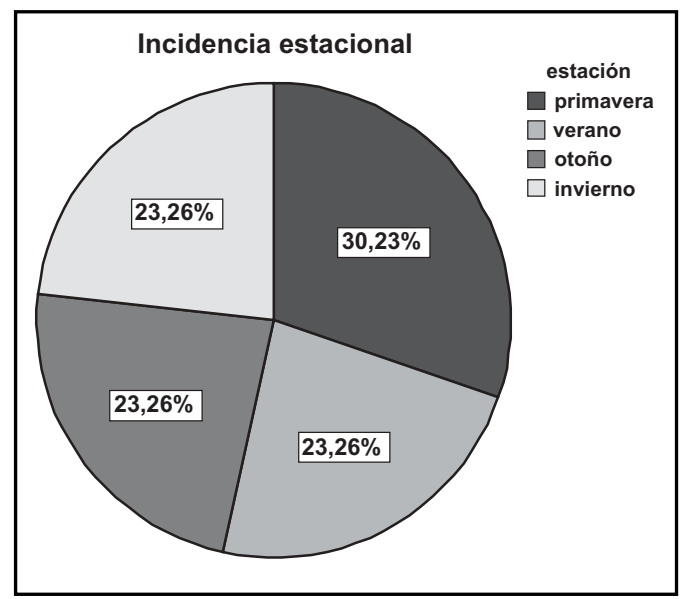

Figura 2. Incidencia estacional de hipoacusia súbita.

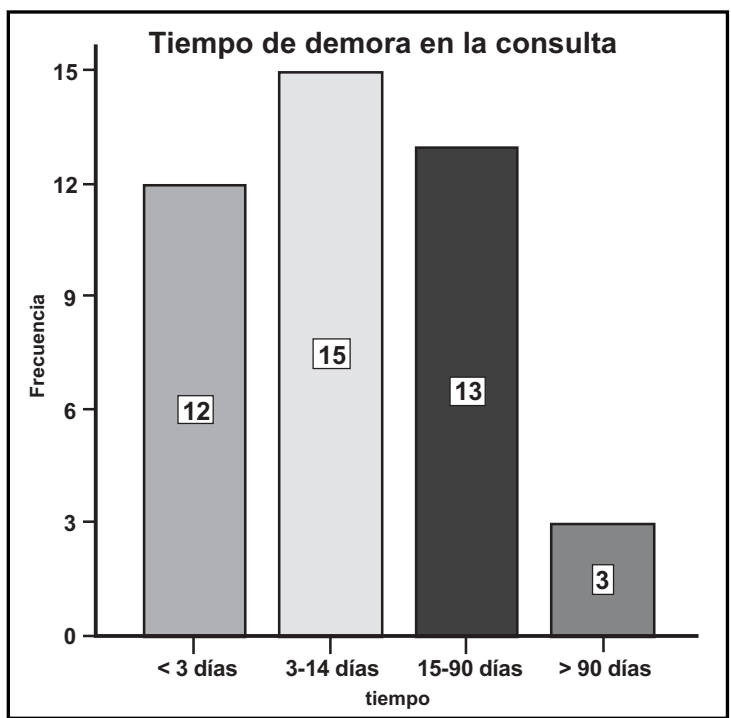

Figura 3. Cantidad de días de demora en consultar versus el número de pacientes. 
Tabla 5. Tratamientos recibidos, se consignan tratamientos coadyuvantes en algunos casos, por lo cual el número de tratamientos es mayor al número de pacientes

\begin{tabular}{|c|c|c|}
\hline Tipo de tratamiento & Fármaco & Número de pacientes \\
\hline Corticoides & Prednisona dosis $1 \mathrm{mg} / \mathrm{kg} / \mathrm{día}$ & 32 \\
\hline \multirow[t]{5}{*}{ Vasodilatadores } & Betahistidina & 2 (coadyuvante) \\
\hline & Nimodipino & 1 \\
\hline & Cinarizina & 3 (2 coadyuvante) \\
\hline & Flunarizina & 3 (coadyuvante) \\
\hline & Pentoxifilina & 1 (coadyuvante) \\
\hline Antiagregantes & Enoxaparina & 1 (coadyuvante) \\
\hline \multirow[t]{5}{*}{ Otros } & Aciclovir & 1 (coadyuvante) \\
\hline & Gingko Biloba & 1 (coadyuvante) \\
\hline & Hidroronol-T & 1 \\
\hline & Ranitidina & 2 \\
\hline & Omeprazol & 2 \\
\hline
\end{tabular}

Tabla 6. Resultados del tratamiento, se aprecia el mejor resultado con las hipoacusias ascendentes y el mal resultado con hipoacusias planas y cofosis

\begin{tabular}{|lccccc|}
\hline & Ascendente & Descendente & Plana & Cofosis & Total \\
\hline No mejora & 4 & 14 & 2 & 11 & 31 \\
Recuperación parcial & 2 & 5 & 1 & 0 & 8 \\
Recuperación total & 4 & 0 & 0 & 0 & 4 \\
\hline
\end{tabular}

consultaron antes de los tres días y el resto de los pacientes.

Al realizar una regresión logística de la mejoría versus las variables: edad, sexo, compromiso OD e inicio antes de los tres días, aparecen significativamente relacionados la edad del paciente con un $\mathrm{OR} 0,9(0,84-0,97)$ y el oído comprometido con un $\mathrm{OR}$ de $0,02(0,001-0,34)(\mathrm{OR}=0$ Odds ratio).

Al analizar la variable "tipo de curva", se observó una asociación con la mejoría ( $p$ value test de Fisher $>0,05$ ), siendo de mal pronóstico las curvas tipo anacusia y descendente, y de buen pronóstico las curvas plana y ascendente.

\section{DISCUSIÓN}

La incidencia de la hipoacusia súbita es de 5 a 20 por 100.000 habitantes, en general bastante baja, llama la atención el número elevado de casos reportados en un año en nuestro hospital (Hospital Clínico de la Universidad de Chile) comparado con otros centros $2,6,19$.

La mayor parte de los pacientes se concentró entre la quinta y séptima década de la vida, lo que es concordante con estudios realizados en el extranjer ${ }^{5}$.
Un grupo pequeño de pacientes presentaron antecedentes mórbidos de importancia, fundamentalmente hipertensión arterial y diabetes mellitus II, que se encuentran generalmente por su alta incidencia en la población que sufre de hipoacusia súbita, de ellos sólo la diabetes mellitus II parece tener relación con la generación del cuadro. Dos pacientes presentaron dislipidemia, uno otitis crónica y uno presentó el antecedente de cirugía prolongada, que han demostrado ser factores de riesgo para sufrir hipoacusia súbita ${ }^{20}$.

Con respecto a los medicamentos que estaban consumiendo los pacientes en el momento del cuadro, no se encontró ninguno asociado a la producción de éste ${ }^{20}$.

Los escasos pacientes que pudieron relatar el inicio de la hipoacusia no tuvieron un predominio horario, sólo 5 lo relacionaron con la mañana que se describe clásicamente como el momento más frecuente de ocurrencia ${ }^{19}$.

Sólo en tres pacientes se pudo relacionar el cuadro de hipoacusia con un cuadro viral previo. No existió un predominio estacional.

El síntoma más frecuente asociado a la hipoacusia fue el tinnitus y el vértigo en concordancia con la literatura ${ }^{19}$. 
La mayor parte de los pacientes tuvo un deterioro auditivo de curva descendente, en menor grado cofosis y "curva plana", esto es concordante con estudios previos².

La mayoría de los pacientes fueron tratados con corticoides (prednisona $1 \mathrm{mg} / \mathrm{kg} /$ día), se usaron vasodilatadores y otros tratamientos coadyuvantes, además hubo un pequeño grupo que por su tiempo de evolución no se les dio tratamiento. No existió un grupo control, por lo tanto, no es posible evaluar la efectividad del tratamiento corticoidal con respecto al total de pacientes.

No se realizó inyección de corticoides intratimpánicos por no estar implementado el procedimiento en nuestro servicio al momento del estudio.

Sólo cerca de un tercio de los pacientes presentó algún grado de recuperación auditiva, pero en nuestra serie la mayoría consultó después de las 72 horas, e incluso meses después de ocurrido el cuadro.

La edad de los pacientes se asoció a la mejoría en forma casi significativa, los pacientes que mejoraron tenían un promedio de edad mucho menor con respecto a los que no mejoraron, esto está acorde a los estudios que muestran que la mayor edad es un signo de mal pronóstico, aunque aún es un tema controvertido $0^{2,35}$.

No existieron diferencias en la probabilidad de mejoría con respecto al sexo de los pacientes.

La mayoría de los pacientes presentaron hipoacusia en el oído izquierdo, esto ha sido reportado anteriormente ${ }^{36}$, por el contrario el hallazgo de un peor pronóstico en la afectación del oído izquierdo no ha sido descrito en la literatura, aunque esta observación debe ser validada por otros estudios.

No se encontró diferencia estadísticamente significativa en la mejoría de los pacientes que consultaron antes de los tres días y el resto de los pacientes, esto es discordante a la literatura clásica sobre el tema y podría deberse a que coincidentemente hubo en este grupo un mayor porcentaje de curvas tipo cofosis que tienen en general pero pronóstico².

En nuestra literatura científica nacional, sólo existe un estudio sobre hipoacusia súbita, en él Correa y cols al igual que en nuestro estudio no demuestran una mayor proporción de pacientes de acuerdo al sexo, o al horario de ocurrencia; en nuestro trabajo se encontró un peor pronóstico en curvas audiométricas que muestran cofosis 0 un patrón descendente, lo cual concuerda con el trabajo previamente nombrado ${ }^{37}$.

\section{CONCLUSION}

La hipoacusia súbita es una patología que aún se nos presenta como un enigma, es importante realizar estudios nacionales prospectivos, en lo posible multicéntricos para validar nuestros hallazgos y el tratamiento de esta patología, incluyendo la inyección intratimpánica de corticoides.

\section{BIBLIOGRAFÍA}

1. De KLEYN A. Suden complete or partial loss of function of the octavus-system in apparently normal persons. Acta Otolaryngol (Stockh) 1944; 32: 407-29.

2. BYL FM. Seventy-six cases of presumed sudden hearing loss occurring in 1973: Prognosis and incidence. Laryngoscope 1977; 87: 817-25.

3. Eisenman D, Arts HA. Effectiveness of treatment for sudden sensorineural hearing loss. Arch Otolaryngol Head Neck Surg 2000; 126: 1161-4.

4. Hughes GB, Freedman MA, Habercamp TH, Guay ME. Sudden sensorineural hearing loss. Cin Aolaryngol 1996; 29(3): 393-405.

5. Schweinfurth JM, Parnes SM, Very M. Current concepts in the diagnosis and treatment of sudden sensorineural hearing loss. Eur Arch Aorhinolaryngol 1996; 253(3): 117-21.

6. Stokroos RJ, Albers FW, Schirm J. Therapy of idiopathic sudden sensorineural hearing loss: antiviral treatment of experimental herpes simplex virus infection of the inner ear. Ann Otol Rhinol Laryngol 1999; 108(5): 423-8.

7. Stokroos RJ, Albers FW, Tenvergert EM. Antiviral treatment of idiopathic sudden sensorineural hearing loss: a prospective, randomized, doubleblind clinical trial. Acta Otolaryngol 1998; 118(4): 488-95.

8. Stokroos RJ, Albers FW, Schirm J. The etiology of idiopathic sudden sensorineural hearing loss. Experimental herpes simplex virus infection of the inner ear. Am J Otol 1998; 19(4): 447-52.

9. SCHUKNeCHT HF, Donovan ED. The pathology of idiopathic sudden sensorineural hearing loss. Arch Aorhinolaryngol 1986; 243(1): 1-15. 
10. Yoon th, Paparella MM, Schachern PA, Alleva M. Histopathology of sudden hearing loss. Laryngoscope 1990; 100(7): 707-15.

11. SchukneCht HF, Igarashi M, Chasin WD. Inner ear hemorrhage in leukemia. A case report. Laryngoscope 1965; 75: 662-8.

12. OGAWA K, KanZAKI J. Aplastic anemia and sudden sensorineural hearing loss. Acta Oolaryngol Suppl 1994; 514: 85-8.

13. Vakkalanka $S$, Ey E, Goldenberg RA. Inner ear hemorrhage and sudden sensorineural hearing Ioss. Am J Otol 2000; 21(5): 764-5.

14. KuzMA BB, Goodman JM. Hemorrhagic lesions causing acute sensorineural hearing loss. Surg Neurol 1998; 49(6): 660-1.

15. García Berrocal J, Ramírez-Camacho R. Sudden sensorineural hearing loss: supporting the inmunologic theory. Ann Otol Rhinol Laryngol 2002; 111: 989-97.

16. OldSTONE MB. Virus-induced autoimmunity: molecular mimicry as a route to autoimmune disease. J Autoimmun 1989; 2 Suppl: 187-94.

17. Stone JH, Francis HW. Immune-mediated inner ear disease. Ourr Opin Rheumatol 2000; 12(1): 32-40.

18. Wu W, Thuomas KA. MR imaging of 495 consecutive cases with sensorineural hearing loss. Acta Radiol 1995; 36(6): 603-9.

19. Cadoni G, Agostino S, Scipione S, Ippolito S, Caselli A, Marchese R, Paludetti G. Sudden sensorineural hearing loss: our experience in diagnosis, treatment, and outcome. $J$ Atolaryngol 2005; 34(6): 395-401.

20. Danielides V, Nousia CS, Bartzokas A, Lolis CJ, KaterI M, Skevas A: Weather conditions and sudden sensorineural hearing loss. BMC Ear Nose Throat Disord 2002; 2: 2.

21. Heiden C, Porzsolt F, Biesinger E, Hoing R. Spontaneous remission of sudden deafness. HNO2000; 48: 621-3.

22. Tamhankar M, Solomon D. Acute hearing loss. Ourr Treat Options Neurol 2004; 6: 55-65.

23. BYL FM, JR. Sudden hearing loss: Eight years' experience and suggested prognostic table. Laryngoscope 1984; 94: 647-61.

24. Ogasamara H, Morimoto K, Ishil K, et al. A sta- tistical analysis of sudden deafness. Nippon Jibiinkoka Gakkai Kaiho 1993; 96: 914-21.

25. Fetterman BL, Saunders JE, Luxford WM. Prognosis and treatment of sudden sensorineural Hearing loss. Am J Ool 1996; 17(4): 529-536.

26. Snow JB, Suga F. Labyrinthine vasodilators. Arch Aolaryngol 1973; 97: 365.

27. Kronenberg J, Almagor M, Bendet E, Kushnir D. Vasoactive therapy versus placebo in the treatment of sudden hearing loss: a double-blind clinical study. Laryngoscope1992; 102(1): 65-8.

28. NaKashima T, Kuno K, YanaGita N. Evaluation of prostaglandin $\mathrm{E} 1$ therapy for sudden deafness. Laryngoscope 1989; 99(5): 542-6.

29. Kalinen J, Laurkanen E, Lappala $P$, Grenman $R$. Sudden deafness: A comparison of anticoagulant therapy and carbogen inhalation therapy. Ann Ool Rhinol Laryngol 1997; 106: 22-6.

30. Kubo T, et AL. Efficacy of defibrinogenation and steroid therapies on sudden deafness. Arch Oolaryngol Head Neck Surg 1988; 114(6): 649-52.

31. WILSON ET AL. The efficacy of steroids in the treatment of idiopathic sudden hearing loss. A double-blind clinical study. Arch Otolaryngol 1980; 106(12): 772-6.

32. Moskowitz D et AL. Steroid use in idiopathic sudden sensorineural hearing loss. Laryngoscope 1984; 94(5 Pt 1): 664-6.

33. Cinamon U, Bendet E, Kronenberg J. Steroids, carbogen or placebo for sudden hearing loss: a prospective double-blind study. Eur Arch Oorhinolaryngol 2001; 258(9): 477-80.

34. WEl ET AL. Steroids for sidiopathic sensorioneural hearing loss. The Cochrane Library, 2006 Issue 3.

35. Mamak A, Yilmaz S, Cansiz H, Inci E, Güçlü E, DeREKöYLü L. A study of prognostics factors in sudden hearing loss. Ear Nose Throat $J$ 2005; 84 (10): 641-4.

36. Zadeh M, Storper I, Spitzer J. Diagnosis and treatment of sudden-onset sensorineural hearing loss: A study of 51 patients. Aolaryngol Head Neck Surg 2003; 128(1): 92-8.

37. A Correa U, A Pacheco T. Hipoacusia Súbita Idiopática. Rev Otorrinolaringol Cir Cabeza Quello 2000; Vol 60: 14-22.

Dirección: Dr. Jorge Zúñiga Pino

Santos Dumont 999 - Independencia

Fono: 9788153 - Fax: 7777338

E mail: jozuniga@med.uchile.cl 